baracken in Karlsruhe fertig gestellt waren, deren Leitung: ihm anvertraut warde. Am 1. April 1871 kehrte or als ordentlicher Professor der Chirurgie nach Dorpat an Adelmanns Stelle zurück, nachdem er einen Ruf nach Froiburg i. Br. abgelehnt und ein solcher nach Königsberg sich zerschlagen hatte. 1877-1878 begleitete er als konsultierender Chirurg die russische Donauarmee beim Uebergange über die Donau, in die Schlachten von Plewna, Felisch und Duback. Nach dem Falle Plewnas, kaum nach Dorpat zurtickgekehrt, traf ihn ein Ruf nach Wiirzburg als Nachfolger Linharts. Zu gleicher Zeit konnte er einen solchen nach Kiew und Petersburg ablehnen. Daranf wurde er 1882 als Nachfolger B. v. Langen becks nach Berlin berufen. : Hier verzichtete er 1884 zum zweiten Male auf die chirurgische Professur in Petersburg. Zu den Titeln eines russischen Staatsrates gesellte sich bald der Adel und die Charakterisierung als Wirklicher Geheimer Rat und Exzellenz. In unserer glorreichen Armee hat or die Stellung eines Generalarztes im Generalleutnantsrange und trägt die schmucke Uniform gern and mit militärischer Straffheit. Sie hat sich in. zwischen mit Orden, Großkreuzen, Sternen mit Brillanten und ohne solche aus aller Herren Ländern und mit bunten, breiten Bändern zu allen Seiten so dicht bedeckt, daß für Nachkömmlinge kaum noch ein Plätzchen zu haben ist. Im Nebenamte wirkt E. v. Berg. mann als Mitglied der Wissenschaftlichen Deputation für das Medizinalwesen und der Kaiser Wilhelms-Akademie für das Militärsanitäts-

\section{Zu E. v. Bergmanns 70. Geburtstage.}

Es wird wohl oft und mit gutem Rechte darüber geklagt, daß unsere Generation zu viel Feste feiert und jede Gelegenheit gern ergreift, um Hurra und Viktoria zu schreien! Das ist unverkennbar ein Zeichen der Dekadenz einer Nation! Wir waren größer und tüchtiger, als wir nur das feierten, was glücklich hinter uns lag oder za einem gesicherten Besitz für uns geworden war. In diesem Lichte erscheinen das goldene Doktorjubiläum und der 70. Geburtstag eines bedeutenden Mannes, zu dem die Welt mit Stolz, Dank und gerechtem Vertrauen emporblickt, als rechte und echte Feste, denn sie sind Höhenstationen, von denen aus man ein der Vollendung nahes, tüchtiges Leben volier Mühen und Arbeit, volles Kämpfe und glücklichen Gelingens, voll Freud und Leid - kurz, ein Leben, das der Psalmist schön nennt, dankbar uberblickt, Ruhepunkte, auf denen man sich all der freundlichen und hohen Gaben, die wir einem solchen Manne verdanken, herzlich erfreuen kann. Dabei drängt sich aber unbemerkt leise in ihren froudenbecher ein herber Tropfen Wermut, die Freude wird durch Leid „getempert und gemischet", wie unsere Vorväter von ihren Rezepturen sagten, mit ejnem schönen Worte: „Sio lächeln unter Tränen", wie die Andromache. Denn die Abendglocken läuten sie vernehmlich ein und klagen mit ihren weichen Harmonien, dab Alles so eitel, ach! so vergänglich ist und das schönste und glücklichste, reichste und fruchtbarste Menschenleben schnell verweht, wie "des Grases Blume auf dem Felde“. Des Wirkens beste theit ist voruber, de Feierabend bricht trüb' und kühl herein. Ist das nicht tranrig? Und doch gibt auch wieder dieser elegische Beigeschmack solchen Festen die hohe Weihe und den intimen Charakter, denn er läßt uns die Gefeierten doppelt wert erscheinen, erhöht den GenuB der köstlichen "Stunden und macht uns in der tröstenden Gewißheit doppelt froh, daß wir noch besitzen, "was so kostlich ist" !

In diesem Sinne wollen wir den 16. Dezember festlich begehen, als den Tag, an dem E. v. Bergmann vor 70 Jahren, um in der Hofsprache zu reden, zur Tafel des Lebens gezogen wurde, und in Dankbarkeit ein Bild von seinem Wesen, Werden und Wirken in den bescheidenen Grenzen, welche der enge Raum einer medizinischen Wochenschrift einer so großen Aufgabe aufzwingt, dem geneigten Leser vorfthren. Wir werden ihm damit nicht viel Neues sagen; da wir aber viel zu bringen haben, so hoffen wir, jedem auch etwas zu bringen, wenn wir sein überaus glückliches, reich bewegtes Leben, in dem der Lorbeerbaum jederzeit neben dem Rosenstrauch stand, vom stillen, heimlichen Winkel eines evangelischen Pfarrhauses in Riga bis zu der hohen Warte, auf die es in Berlin geführt ist, an uns voruberziehen lassen.

E. v. Bergmann studierte in Dorpat von 1854-1860, promovierte daselbst am 13 . November 1860 mit der Dissertation: De balsamo copaivae cabebarumque in urinae transito, trat dann gleich bei Adelmann, seinem künftigen Schwiegervater, und bei Oettingen als Assistent an der Chirurgischen Khnik ein und habilitierte sich dort auch mit der Schrift: „Ueber Fettembolie“ 1864 als Privatdozent der Chirurgie. Nach einer Studienreise nach Berlin und Wien ging er mit dem konsultierenden Generalarzt Prof. Dr. Wagner als Assistent auf den Kriegsschauplatz in Böhmen und nach dem Friedensschlușse nach Dorpat zurück. ' 1870 übernahm er erst unter Volkmanns, dann unter Billroths Leitung das Reservelazarett Seilebohn in Mannheim, fuhr später mit dem badischen Sanitätszuge nach Raon, Belfort und in die Lazarette vor dem belagerten Paris, bis die Friedrichs- wesen. Durch besonderes Vertrauen des Kaisers wurde er auch Mitglied des Herrenhauses.

So ist sein heller Stern in mächtig schnellem Zuge wie ein Meteor am chirurgischen Firmament emporgéstiegen. Das verdankt E. v. Bergmann in erster Linie seiner großen uud erfolgreichen literarisch en Tutigkeit. Es erlaubt uns nicht der Raum, hier auf eine eingehende Schilderung seiner schriftstellerischen Verdienste einzugehen; damit würden wir auch Enlen nach Athen tragen, denn sie sind, wie unsere Juristen sprechen würden, weltkundig. Ein Gesamtüberblick über sie zeigt, da 3 sich seine Arbeiten auf drei Gebieten mit besonderem Erfolge und der ihm eigenen Grindlichkejt betätigt haben.

1. Als E. v. Bergmann eintrat in die klinische Hospitalpraxis, herrschten Pyämie, Sepsis, Hospitalbrand und Rose verheerend wie mörderische Seuchen, unausrottbar und täglich die schmerzlichsten Opfer fordernd, bis zur Vernichtung jeder operativen und chirurgischen Tátigkeit in den Krankensälen. Panaritien und Furunkel führten zum Tode, keine Amputation eines größeren Gliedes glückte. Es war eine chirurgische Praxis zum Gotts Erbarmen. Alles seufzte nach Errlösung! Nur Jüngken, den man bei 40 Jahren schon den „alten" nannte, obwohl er sich als Prophet der neuesten Chirurgie ausgab, tröstete sich, daß es doch kein Wunder sei, daß das ,lymphatisch venöse und skrophulöse Geschlecht den atmosphärisch-tellurischen Einflüssen bei Jerwundungen und Operationen erläge ${ }^{*} !$ Die Hospitalbrandigen versteejze er, nachdem er mit dem Glüheisen in ihren Wunden heramgefalien war, sorgfaltig. Stromeyer nante dalier die Seuche in der Charité: Geheimen Ober-Hospitalbrand.

Der jugendliche Forscher E. $\nabla$. Bergmann betrachtete es dagegen mit Recht als die dringendste Aufgabe, die chemische Natur des septischen Giftes und den Vorgang, auch die Zoichen der putriden lnfektion zu ergründen. Es war bei den mangelhaften Instrumenten und den unzureichenden Methoden, auch auf den eingeschlagenen Wegen, wie man jetzt einsieht, nicht möglich, die gestellten Fragen zu lösen, doch bleiben die experimentellen uud klinischen Beobachtungen, die E. v. Bergmann dabei erzielte, von dauerndem Werte: „Ueber das septische Fieber 1868. Ueber das putride Gift 1868 (in Gemeinschaft mit Sch miedeberg). Ueber das Verhältnis der Fermentintoxikation zur Septicämie 1882 (in Gemeinschaft mit Angerer). Die Gruppierung der Wundkrankheiten 1882.“

Als nun 1870 die Antisepsis Listers, wie die Sonne am Oster. morgen, Alles erwärmend, erleuchtend, erlösend und neues Leben, frischen Mut erweckend in den Krankensälen, und damit die Glanzzeit der Chirurgie aufging, trat auch gleich E. v. Bergmann als tief grabender Arbeiter auf dem fruchtbaren Felde voll frischen Erdgeruchs auf und führte das bis zur Stunde wirksamste Antisepticum, das Sublimat, in die Chirurgie ein. Immer mehr ging er aus der Antisepsis in die A sepsis über, bis sein Assistent Schimmelbusch diese als ein fertiges Verfahren in den Werken: "Die Durchführung der Asepsis in der v. Bergmannschen Klinik" "(Arbeiten der chirurgischen Klinik 5), "Anleitung zur aseptischen Wundbehandlung" 1892, "Desinfektion septisch infizierter Wunden“ 1895 , darstellen konnte. Sie hat sich zurzeit allgemeine Giltigkeit in Kriegs: und Friedenszeiten in aller Welt erworben und reiht sich Listers bahnbrechenden Arbeiten würdig als SchluBstein und unvergängliches Verdienst E. v. Bergmanns an.

2. In den reiferen Jahren seines Schaffens wandte sich E. v. Bergmann der Hirnchirurgie zu. Sie war damals ein Brachfeld. Niemand wagte in der vorantiseptischen Zeit mit operativen 
Eingriften in die Schădelhơhle einzudringen, da von den Schthdelverletzten weaige genasen. Die Trepanation war so verschrien, dak Stromeyer als erste Indikation für sie aufstellte, daß der Chirurg rorher auf den Kopf gefalien sein müse. J in ngken orklarte eine I'repanation, die ich vorgenommen hatte, in der Klinik filr ein Verbrechen. Frst die Antisepsis und die groben Fatdeckungen der Hirnlokalisationen durch Hitzig und Fritsch, Broca, Horsley und andere eroberten das heute so fruchtbare, bluthende Feld der Hirnchturgie, und E. v. Bergmann ging uns dabei mit Besonnenhejt und Kühnheit voran. Er sicherte durch seine Arbeiten die Diagrose und verbesserte rnit besonderen Glück die Operationsmethoden: "Die Lehre von den Kopfvertetzungen" 1973 und 1880, Die Diagnose der traumatischen Meningitis" 1876 , "Die chirurgische Behandlung der Firnkrankheiten" in drei Auflugen. "Die chirurgische Behandlung der Gehirngeschwitlste $\theta^{\mu}$, im ergten Bande des Handbuehs der praktischen Chirurgie, in zwei Auflagen. "Firnverletaungen mit allgemeinen und I lerdsymptomen" $18 B \mathrm{~B}$. Die chirurgische Behandlung der Gehirngeschwälste".

Mit diesen Arbeiten begrindete F. v. Bergmann einen der wesentlichsten Fortschritte der modernen

Chirurgie. Sie sind der duftigste Zweig in seinem Ruhmeskranze!

3. Zuletzet brachte er axch die Frachte heim, die îm wăhrend der Kriege auf den Schlachtfeldern und in dea Laparctten unter dem Donner der Geschütze und im Angesichte des T'odes erwachsen waren. Sie sind nicht minder reif mad kostbar, als die schon erwathraten: "Die Resultate der Gelenkresektionen im Kriege" 1874. "Schufwunden und Unterbindungen der Subelavis" 1877. Behandlung der Schubverletzunizen des Kniegelenkes" 1878. .Deber das Schicksal der Trangfusion" 188\%. "Einheilang des" Kugeln in Hirn nebat Behandlung der Schubwrunden im Kriege" 1895. "Zwei Vorthrage liber die erste Hilfo bei BchnBverlatzangen im Kriege" 1902. "Die Schulsverletzungen im Frieden ${ }^{*} 190 \%$.

Das wesentlichste Verdienst $\nabla$. Be r mans anf diesen Feldo ist die Einfuhruag der aseptischen Occlusion der Schubwandew, einer Methode, die sich in allon modernen Kriegen, begonders in derl langgezoginen, blutigen Schlachten des rassisch-japanigchen, bewhit und ein unvergkngliches Burger recht in der Kriegschirurgie erobert hat.

Daneben gibt es aber fast kein Qebiet der Chirurgie, das E. v. Bergmann vicht nut seinen Benbrchtungen und forschungen geklärt, erhellt und befruchtet bätte. Da ron geben zahlreiche Artikel von ihun in

Zeitschriften und Archiven Kunde. - Alle geine Arboiten zeichnen sich aus durch die experimentelle Richtung, deren Methoden und Mittel er sicher beherrschte. Sie war aus der Chirurgie ganz verschwunder. Dio enormen. Fortschritte, weiehe die allgemeine Chir trigie gemacht hat, verdanken wir unbestreitbar dem Experimente, wean auch die praktischen bifolge schon oft seinen Ergebnissen vorsus geoilt waren. Dazk kam E, v. Bergmanns tuchtigo physiologische und chemische Vorbildung, sein reiches Wissen in iler allgemeinen Pathologie und pathologischen Anatomie, scin sicheres Beherrsehen der klinischen Untersuchungsmethoden, seine genaue Literaturkenntnis und gefibtes Beobachtnngstialent. Bedenkt man dabei, daß E. $\nabla$. Bergmann Faupt- oiler Mitredak. teur von Langenbecks Archiv, vom Zentrablabt für Chirurgio von Volkmanns Fortrigen, von der groBartig angelegten ind groszutgig durchgefuhrten Dentachen Chirurgio, ron dem Itandbuche der pralktischen Chiruryie etc. war, dafl er zeitraubende fotachten und Revisionen in der Wissenschaftlichen Deputation und Akademie anfertigen mugte, so fragt man erstannt, woher hat er die Zeit genommen? Alles ist mit weitem Blick and freier Auffassang entworfen, tief und grindlich durchdacht, allerorten das Detail den groBen Gosichtspunkten unteryeordnet, alles geistroll, anziehend, nicht ahne poetischen Hauch geschrieben. Kurz, die bedeutendsten und ergebnisreichsten Werke der chirurgischen Literatur! Ueberall tritt seine Pioniernatur hervor, die sich wohl irren konnte, aber immer anregend wirkte. Fremd war inm eine muhselige, durltige Nachlese hinter dem Ermtewagen, er sehnitt frisch und froh anf jungfralizhem Felde. Mit Zähigkeit waßte er seine Arbeiten gegen Sturm und Minen wie eine Festung za rerteidigen, doch hielt er sich stets in angenehmen Formen und bei guten Sitten in der Entgegnung. war anch gern bareit, Irrttmer zuzugestehen und AuBen- werke ohne Zaudern aufzugeben. - Bei allen medizinighen Konpressen, der Berlinor mediainischen Gesellschaft, den chirurgischen Vereinen und dem Kongrel der Deutschen Gesellschaft für Chirurgie ist er Mitglied und fehlt selten bei ihren Tagnngen auch in den entferntesten Ländern. Wurch Vortrage, führende und kritische Bemerkungen in den Diskussionen wubte er Leben und Inhalt in ihre Verthandlungen zil bringen. Was er sagte, war stets klug, wahr, anregend und belekrend, da er aus dem reiehen Born seiner Stadien und Exfahrungen schopfte. Auch an bffentlichen Vorträgen beteiligte er sich gern. Das lebendigo Wort, das beweglicbste Hiffs" mittel des menschlichen Geistes, steht ifum willig zurs Verfügrng er ist ein Mann von hinreißender Beredtzankeit! So bekam er bald die Führung in die Hand. Die Gesellschaften. Kongresse und Vereine wallten ihn zu ihrem Vorsityenden, nachdem er jhen mit Treue und Hingebung gedient hatte. Wie viel verdankt thm die Deutsche Geselischaft für Chirurgie! Er hat ihr in Langenbeek . hause ein köstliches Heim geschaffen, das er mit besonderer Liebe wohnlich erhait, ihren Wohlstand und Weltruf begrilndet und ihre Tayungen zu Weltereignissen gemacht. Wie versteht er es, in der Berliner medizinischen Gesellschaft dem guten Ton und die wissenschaftiche Leistung zu fördera! Woljin wir blicken, Firnealicht, dass grobe, stille Tuenchten! Wir fragen verwundert, wie hat er das gemacht? Nun! Vor den Erfolg und die Tugend setzten die Götter den GchweiB! E. v. Bergmann hat die Hände fleißig geribrt und die Minuten, deren jede cine gebielerische Forderung brachte, auszurutzen verstanden. Sejue durchwachten Nächte haben un erc Tage erhellt! Doch merkt man es auch seiner temperawentvollen, sprudeluden Art im Schreiben an, dals ihm die Tinte leicht und anmutig ats der Fetler fliebt, Eine onorus Arboitakraft stelit bei illm mit einer elstaunlichen Arbeitslust im schönen Butede

Das Schicksal hat F. v. Bergmano anf hohe, stark besuchte Lehykanzeln ge stellt! Aus ihren gesegneten Hörsälen hat sich ein maditizer Strom auserltsenen Wissens und veredelnder Bildung in Lanfe der Jahrhuuderte iber die wissensdurstige Ingrend ergossen. Welche IReihe edler Männer und turbtiger Forscher haben hier gewirkt und sind ans ihnen hervorgegangen! Znletzt ist es ihm vergöunt, als Nachfolger der grobten Chirurgen auf ejner Stelle zu wirken, an der Dieffenbach, der $\mathrm{Er}$ lanchtesten einer, begaadigt war, im Angc sirhts seiner Zuhborer, das lebendige Wort auf den beredten Lippen, in der lcanstgetibten Hand das Yesser. den be neidensworten Tod za sterben. Uad wie hat L v Bermman seine schwere Aufgabe, es solchen Vorgängern gleich au tun, gelöst! Wie gokrates von seinem Lehramte, so sprach auch er: denn also bofiehlt mir der Gott, das wisset wohl'* Der tiefe, sittliche Ernst, die unerbittliche Wahrheitsliebe, lite eingehende kritische Wirdigung der Literatur und dabei ein immer wieder durchbrechender, orwärmender Humor machen scine Lchimethode den Schülern unersetzlich. Das großartige Material, welches die Klinik darbietet, wird sorglälig fïr die Lehrawecke verwendet. In der Fithrung des Yfossers ist er seinen großen Amtsworgängern alle Zeit gewachsen gewesen. Sein Grandsatz ist, kejne Oparation zuzalssaten, die wicht einen gaten Erfolg for den Kranken verheibt, einer dringenden Indikation entspricht. Und wic reich an Trost nnd heralichem Erbarmen ist er seinen Patienten gegenüber, seien sie aus der elendesten Hutte oder ans stolzen schlossern und müchtigon Burgen! Er ist ein ge borener Arzt und deshalb anch gesucht ron deu Großen der Erde wie von den Miihseligen und Beladenen im Folke! Selbst die l'fortien des wohl verwabiten Harens des Grofmoguls kaben sich ihm erschlossen! Von alle Erdteilen strömen za ihm Filfesuchende toder erschallt an itto der liuf zu kommen. Darum feiert heute vicle Tausende ungesehen mit uast Wir wollen nns auch dankbsy erinnern, daB er den Liebling unseres Volkes, den schwer heimgesuchten Kaiser Fiedrich auf seinem schmerzensreichen Krankenlager trotz des hathlichen und verletzenden Benehmens seines engliselzen Kollegen xait unersehöpflicher Geduld und erbarmender Liebe gepflegt und unseren jetzigen Kaiser glïcklich operiert und gesund erhalten hat! Man sagt wohl, daß ein Mensch mit grol3em Selbstbewußtwein nicht ungestraft unter Palmen wandelt und grobe Männer leieldt ihre inzere Festigkeit im Schatten matuhtiger Hermoler verieres. Das lat 
E. v. Bergmann nie getan, Männerstolz vor Königsthronen war seines Lebens Zierde!

Wie die Harmonie eines edlen Menschenlebens im rechten Verhältnis zwischen Kopf and Herz, Verstand und Wille, Intelligenz únd homaner Gesinnung besteht, so gët bei E. v. Bergmann die geistige Größe mit einer erquickenden Herzensgüte eibher tind verleiht seinem Wesen den persönlichen Zauber, der alle bundigt, die mit ihm veykehren. In der Grazie der beiteren Lebensführung, in bohen Sinne der Auffassung und Bewältigung der Lebensaufgaben steht er vor uns als einc vornehme Persönlichkeit, mit offenem Ange und jugendlichem Herzen alle Gebiete des Lebens überschanend und beherrschend, ehrlich, wahrluaftig, zuverlässig, treu und hingebend in der Familie wie in der Freundschaft. Alle freudigen und traurigen Ereignisse in der Familie der Aerzte und Freunde finden in seinem weichen Gemüte ein ungefürbtes Echo. Er übt die schwere Kunst des Vergebens und Vergessens! Das Wohl und Fortkommen seiner Assistenten liegt ihm am Herzen, als von Gliedern seiner Familie. Den leider so früh dahingeschiedenen N a sse hat er begraben und betrauert wie einen Sohn. Mit Geduld und Langmut behandelt er seine Untergebenen bis zum letzten Diener in der Klinik und versteht sie gut zu versorgen. Den Arbeiten der jüngeren Generation weil乃 er mit Interesse zu folgen und sie zu fördern! Bei aller Güte und Milde zeigt er sich aber doch in allen Lagen und bei allen Aufgaben als ein stahlharter, ganz in sich rubender Charakter, zähe, aufrecht und voll straffer Énergie, der im Donner der Schlachten ruhig, im Dienste unerbittlich vor keinem Hindernis zurückschreckt - mit einem Worte als eine echte Fübrernatur. Er besitzt den Trieb zur Tat, und alle guten Mittel, die zum Ziele führen, weiß er auch für seine Absichten mobil zu machen. Fin Weichen und Wanken, ein Zögern ind Schwanken kennt er nicht!

Wer an der reichen ind doch so gemütlichen Geselligkeit seines alle Zeit offenen und hoch gefïhrten Hauses, in dem eine gleichgestimmte, feinsinnige Gattin mit fesselnder Grazie und herzgewinnender Freundlichkeit ohne Zwang weit tiber hundert Gäste angenehm zu bewirten versteht, teilgenommen hat, wird den warmen Hauch der liebenswürdigen Gemütlichkeit und den von herzgewinnendem Humor durchwehten Frohsinn, der von dem Wirte ausgeht, noch lange dankbar nachempfinden.

So sichert E. v. Bergmann ein tüchtiges Schaffen einen ehrenvollen Platz in der Geschichte der Chirurgie und seiner Sitten Freundlichkeit die dankbare Ergebenheit und Verehrung seiner Freunde. Er hat nach Goethes schönem Worte das höchste Glück der Eruenkinder, die volle Entfaltung der Persönlichkeit erreicht! Und von den großen Persönlichkeiten hängt der Fortschritt der Menschheit ab! Nun! Gott Lob! Er sitzt heute nicht auf die Postille gebückt zur Seite des wärmenden Ofens, nein! stattlich und aufrecht. kräftig und frei steht er vor uns in frischem Wirken und voll frohen Lebens!

Bringt denn Eichenlaub und Palmen;

Aus dem Garten, von den Almen

Blumen, die das Aug' entzücken,

Um zum Kranze sie zu winden,

Unserm Freund die Stirn zu schmücken,

Auch den Wunsch hineinzubinden,

$\mathrm{Daf}$ er lange noch voll $\mathrm{Kraft}$

Jugendfrische Werke schafft,

Und, wie's Abraham erfahren,

Sanft beimfahre, "satt an Jahren"!

H. Fischer (Breslau-Berlin). 